\title{
Effects of problem size, operation, and working-memory span on simple-arithmetic strategies: differences between children and adults?
}

\author{
Ineke Imbo • André Vandierendonck
}

Received: 4 October 2006 / Accepted: 20 February 2007 / Published online: 25 April 2007

(c) Springer-Verlag 2007

\begin{abstract}
Adult's simple-arithmetic strategy use depends on problem-related characteristics, such as problem size and operation, and on individual-difference variables, such as working-memory span. The current study investigates (a) whether the effects of problem size, operation, and working-memory span on children's simple-arithmetic strategy use are equal to those observed in adults, and (b) how these effects emerge and change across age. To this end, simple-arithmetic performance measures and a working-memory span measure were obtained from 8-year-old, 10-year-old, and 12-year-old children. Results showed that the problem-size effect in children results from the same strategic performance differences as in adults (i.e., sizerelated differences in strategy selection, retrieval efficiency, and procedural efficiency). Operation-related effects in children were equal to those observed in adults as well, with more frequent retrieval use on multiplication, more efficient strategy execution in addition, and more pronounced changes in multiplication. Finally, the advantage of having a large working-memory span was also present in children. The differences and similarities across children's and adult's strategic performance and the relevance of arithmetic models are discussed.
\end{abstract}

\section{Introduction}

The study of arithmetic performance is an important topic, since children spend a great deal of time mastering this skill

I. Imbo $(\bowtie) \cdot$ A. Vandierendonck

Department of Experimental Psychology,

Ghent University, Henri Dunantlaan 2,

9000 Ghent, Belgium

e-mail: Ineke.Imbo@UGent.be and adults continue to use it in daily life. A well-documented observation is that a number of different strategies are used by adults as well as children to solve simple-arithmetic problems. Performance on a problem depends on both strategy selection and strategy efficiency. Strategy selection refers to the choice of a strategy among a set of alternatives available to solve the problem. In the domain of mental arithmetic, direct memory retrieval is distinguished from procedural strategies ${ }^{1}$ such as counting (e.g., $7+4=7 \ldots 8 \ldots 9 \ldots 10 \ldots 11 ; 3 \times 7=7 \ldots 14 \ldots 21)$ and transformation (e.g., $8+5=8+2+3 ; 9 \times 6=10 \times 6-6$ ). Strategy efficiency refers to how fast and accurate strategies lead to the solution. Retrieval is generally more efficient than transformation, which is still more efficient than counting.

Accurate information about which strategies are applied (strategy selection) and how the strategies are applied (strategy efficiency) can be obtained by the combination of two approaches of data collection-self-reports and response latencies (Hopkins \& Lawson, 2002). More precisely, in such a combined approach, trials are first separated by self-reports and then response latencies are analyzed. Using this combination of approaches, it has been shown that adult's strategic performance is influenced by both problem-related characteristics (such as operation and problem size) as well as by individual-difference variables (such as working-memory span). In the current study, we investigate whether or not children's strategic performance is influenced by the same variables. Moreover, we also

\footnotetext{
${ }^{1}$ Many different labels have been used to denote what we call here 'procedural' strategies. Examples are "reconstructive strategies", "algorithmic strategies", "back-up strategies", "the usage of manipulatives", et cetera. In the current study, we consistently use the term "procedural' strategies, to refer to (mostly time-consuming) strategies in which the solution is obtained in a sequence of operations.
} 
aimed at examining how the influence of these variables emerges and changes across primary school years, and whether these changes can be compared with practice or training effects in adults.

\section{Effects of problem size on arithmetic strategy use}

The problem-size effect, which refers to slower and more error-prone performance on large problems (e.g., $8 \times 9$ ) than on small problems (e.g., $2 \times 3$ ), is one of the most robust effects observed in mental-arithmetic research (Ashcraft 1992; Zbrodoff 1995). According to Campbell and Xue (2001), there are three strategy-related sources of the problem-size effect in adults: less frequent retrieval use for large than for small problems, lower retrieval efficiency for large than for small problems, and lower procedural efficiency for large than for small problems. In the current study, we investigated which of these sources determine the problem-size effect in children. More specifically, we checked for three different age groups (i.e., 8-, 10-, and 12year olds) whether the problem-size effect was significant in terms of strategy selection (i.e., more frequent retrieval use on small than on large problems) and in terms of strategy efficiency (i.e., more efficient retrieval and procedural use on small than on large problems).

We also investigated whether the contribution of the different sources of the problem-size effect changes across the primary school years. Chronometric-only studies (i.e., without strategy reports) showed that the problem-size effect decreases gradually with age (e.g., Campbell \& Graham, 1985; Cooney, Swanson, \& Ladd, 1988; De Brauwer, Verguts, \& Fias, 2006; Koshmider \& Ashcraft, 1991). However, no study thus far used the combined approach (i.e., collecting self-reports and response latencies) to investigate which strategic sources contribute to the agerelated decrease in the problem-size effect. We expected that age-related increases in retrieval use, retrieval efficiency, and procedural efficiency would be larger for large problems than for small problems (i.e., an age by size interaction).

Effects of operation on arithmetic strategy use

Adult studies consistently show operation-related differences in both strategy selection and strategy efficiency (e.g., Campbell, 1995; Campbell \& Xue, 2001; Hecht, 1999; Imbo, Vandierendonck, \& Rosseel, 2007, in press). Generally, retrieval is used more frequently in multiplication than in addition, whereas both retrieval and procedural efficiencies are higher in addition than in multiplication. The joint investigation of children's performance in addition and multiplication is rather scarce, however. Lépine, Roussel, and Fayol (2003) investigated 5th graders' addition and multiplication verification performance (e.g., $2+3=7$, true/false?). Although children did not have to report which strategies they used, Lépine et al. (2003) used priming techniques to infer which strategies the children used. Based on the observation that priming the operation sign $(+$ or $x)$ reduced addition response times but not multiplication response times, they inferred that addition problems were generally solved by means of procedures whereas multiplication problems were rather solved by direct fact retrieval. To test whether this operation-dependent effect on strategy selection changes across age, Lépine et al. (2003) compared their results with those obtained by Roussel, Fayol, and Barrouillet (2002), who tested the same verification problems in adults. Apart from faster response times in adults than in 5th graders, similar effects occurred in both age groups. Consequently, Lépine et al. (2003) conclude that addition and multiplication problems are solved similarly by fifth graders and adults, i.e., by means of procedural and retrieval strategies, respectively.

The current study aimed to test (a) at what age these operation-related differences originate, and (b) whether or not these operation-related differences change across age. In contrast to Lépine et al. (2003), who used a verification task without strategy reports, we used a production task with trial-by-trial strategy reports. Moreover, we tested three different age groups (second, fourth, and sixth graders) whereas Lépine et al. tested fifth graders only. The retrieval bias for multiplication over addition was expected to originate from second grade on. Indeed, as addition is taught already in first grade, children in our study (second, fourth, and sixth graders) should master this operation reasonably well. As multiplication is taught in second grade, the youngest children of our study were only starting to master this operation. Because multiplication performance is strongly based on direct fact retrieval, we expected larger increases in retrieval use for multiplication than for addition, and especially between second and fourth grade. From fourth grade on, we expected to observe the same operation-related differences in children as in adults; i.e., more frequent retrieval use in multiplication than in addition and more efficient strategy execution in addition than in multiplication.

Effects of working-memory span on arithmetic strategy use

It has been shown that working memory, a memory system involved in concurrent maintenance and processing of information (Baddeley, 1996; Baddeley \& Logie, 1999), plays a significant role in adults' arithmetic performance (see DeStefano \& LeFevre, 2004, for a review on dual-task studies). Low-span adults have been shown to perform worse on arithmetic tasks than high-span adults (e.g., Jurden, 1995; Seyler, Kirk, \& Ashcraft, 2003), but it is not 
known whether this effect is due to individual differences in strategy selection, strategy efficiency, or both. Working memory in children has been studied in relation to mathematical disabilities (see Geary, 2004, for a review); rather than in relation to its role in normally developing children. As respects strategy selection, higher working-memory spans have been linked with less frequent use of procedural strategies and more frequent use of retrieval strategies (e.g., Barrouillet \& Lépine, 2005; Geary, Bow-Thomas, Liu, \& Siegler, 1996; Geary, Hoard, Byrd-Craven, \&, DeSoto, 2004; Noël, Seron, \& Trovarelli, 2004; Steel \& Funnel, 2001). Working memory has also been related to strategy efficiency. Adams and Hitch (1997) observed faster arithmetic performance in children with higher working-memory spans. Since no strategy reports were obtained, it is not clear whether working-memory span was correlated with both retrieval and procedural efficiency. Rasmussen and Bisanz (2005) observed that several working-memory measures together accounted for a substantial proportion of the variability in arithmetic performance $\left(R^{2} \geq 0.40\right)$. Comparable results were obtained by Swanson (2004; Swanson \& Beebe-Frankenberger, 2004), who showed that working memory predicted solution accuracy of arithmetic word problems independent of other skill measures such as fluid intelligence, reading skill, math skill, and short-term memory. Comparably, Noël et al. (2004) observed that children's addition accuracy was not predicted by processing speed but that it was predicted by several measures of working-memory capacity. Finally, Barrouillet and Lépine (2005) observed that direct memory retrieval was faster in high-span children than in low-span children.

The current study investigated (a) whether workingmemory capacity is differently correlated with children's strategy selection and strategy efficiency, and (b) whether the importance of having a large working-memory capacity stays equally important throughout the primary school years. Several predictions were made. Problem-answer associations in long-term memory can only be strengthened if both the problem and the answer are hold simultaneously active in working memory (e.g., Geary, 1993, 1994). Hence, poor working-memory resources may result in weaker and less accessible associations in long-term memory. Based on this reasoning, we predict more frequent retrieval use in high-span children than in low-span children. Second, since poor working-memory resources may lead to smaller amounts of available attentional resources, we predict more efficient retrieval use and more efficient procedural use in high-span children than in low-span children. Indeed, attentional resources are needed to activate items in long-term memory and to maintain this activation (Barrouillet, Bernardin, \& Camos, 2004; Lovett, Reder, \& Lebière, 1999) and to execute several subprocesses needed in procedural strategies (DeStefano \& LeFevre, 2004).
Finally, we hypothesized that the advantage of having a large working-memory capacity would decrease with age. The latter prediction was based on previous findings arguing that cognitive resources are most important during the initial phase of skill acquisition whereas their role declines as facts become represented in long-term memory (e.g., Ackerman, 1988).

\section{Simple-arithmetic models}

Finally, we also wanted to test whether arithmetic models are able to explain our results. One model that is especially relevant in the present context is the adaptive strategy choice model (ASCM) of Siegler and Shipley (1995). In this model, people have several strategies available. When encountered with a simple-arithmetic problem, they will try to choose the fastest and most accurate strategy among all available strategies. However, people also set a confidence criterion, which determines how sure they must be to state a retrieved answer, and a search length, which determines how many attempts they will make to retrieve an answer before trying a procedural strategy to solve the problem. One will thus retrieve the answer from long-term memory only if the problem can be solved fast and accurately with the retrieval strategy. Otherwise stated, the retrieval time may not exceed the search length criterion whereas the confidence criterion should be exceeded. If the retrieval strategy would provide a slow and/or incorrect answer (e.g., when a problem is associated with several possible answers in long-term memory) and thus exceeds the search length or does not exceed the confidence criterion, one will rather use a procedural strategy to solve the problem.

The ASCM also predicts the efficiency with which retrieval and procedural strategies will be executed. The efficiency of retrieval strategies depends on the number of searches in long-term memory. If the distribution of problem-answer associations is peaked (i.e., only one answer receives high activation), the correct answer will be retrieved very fast. If the distribution of problem-answer associations is flat (i.e., many answers receive activation), more time is needed to search the correct answer among several incorrect (but highly related) answers, which have to be inhibited. The efficiency of procedural strategies, in contrast, does not depend on the peakedness of problemanswer associations but on the difficulty of executing the particular procedural strategy. For example, the number of counts determines the efficiency of the counting strategy. In the current study, we explicitly tested this prediction of Siegler's model. More specifically, because both retrieval frequency and retrieval efficiency rely on the peakedness of problem-answer associations, it was hypothesized that retrieval frequency would be highly correlated with retrieval efficiency. However, because procedural efficiency 
does not rely on the distribution of problem-answer associations but rather on the number of steps to be executed, procedural frequency, or its component, retrieval frequency, should not be correlated with procedural efficiency.

\section{The present study}

To summarize, the purpose of the present study was to test whether the effects of problem size, operation, and working-memory span observed in children are similar to those observed in adults. We also wanted to test whether agerelated effects in children can be compared with practice effects in adults. Several predictions were made. Since the magnitude of the problem-size effect decreases with age, we expected that the age-related increase in retrieval use and strategy efficiency would be larger for large problems than for small problems (i.e., an age by size interaction). Because multiplication procedures are more difficult to execute in comparison to addition procedures, children might be more motivated to remember multiplication facts. We thus expected larger increases in retrieval use and strategy efficiency for multiplication than for addition, and especially between second and fourth grade (i.e., an age by operation interaction). Finally, we hypothesized that having a large working-memory capacity would correlate with more frequent retrieval use and more efficient strategy execution. However, the advantages of having a large workingmemory capacity were expected to decrease with age.

\section{Method}

\section{Participants}

Sixty children participated. They all attended the same elementary school in the Flemish part of Belgium. Twenty of them were in the second grade of elementary school (mean age: 8 years 0 months; 9 girls and 11 boys), twenty other children were in the fourth grade of elementary school (mean age: 10 years 0 months; 10 girls and 10 boys), and the last twenty children were in the sixth grade of elementary school (mean age: 12 years 0 months; 7 girls and 13 boys). The children were selected from the whole ability range, although those who were considered by their teachers to have specific learning or behavioral difficulties were excluded. The children had no documented brain injury, socio-cultural disadvantage, or behavioral problems. The children only participated when they, as well as their teachers and their parents consented.

To verify whether the three age groups were representative samples of the population, a standardized skill test [Arithmetic Tempo Test (ATT), De Vos, 1992] was administered. This pen-and-paper test consists of several subtests that require very elementary computations (e.g., $2+3=$ ?). Each subtest concerns only one arithmetic operation (addition, subtraction, multiplication, or division). In the present experiment, the first two subtests were administered, i.e., the addition and the subtraction subtest, each consisting of 40 items of increasing difficulty. We opted for these operations since the problems of the multiplication and division subtest were beyond the second graders' skill (e.g., $12 \times 4$, 75:25). The children were given 1 minute for each subtest and had to solve as many problems as possible within that minute. Performance on the test was the sum of both subtests. An ANOVA on these performance data with grade (2, $4,6)$ as between-subjects factor showed a main effect of grade, $F(2,57)=61.6$, with increasing performance across the second, fourth, and sixth grade (scores of 29.4, 48.3, and 55.9, respectively). We further tested whether the children differed from the expected ATT performance. Therefore, the score expected at the moment of testing (i.e., 19 educational months for second graders, 39 educational months for fourth graders, and 59 educational months for sixth graders) was compared with each child's individual score. Paired-samples $t$ tests (two-tailed) showed no significant differences between observed and expected ATT performance, with $t$ values of $0.4,1.9$, and 1.7 for second, fourth, and sixth graders respectively (all $P \mathrm{~s}>0.05$ ). Clearly, the three age groups were representative subgroups of the population.

\section{Materials and procedure}

The children were individually tested in the month of May. At that moment, even the second graders had learned how to solve simple addition problems (up to 20) and simple multiplication problems (up to 100). All children were administered a simple-arithmetic task in which they had to solve addition and multiplication problems, and a readingspan task to test their complex working-memory span.

\section{Simple-arithmetic task}

All children solved 56 simple addition problems and 56 simple multiplication problems. The problems were constructed from all the possible pair-wise combinations of the integers $2-9$ with tie problems (e.g., $2+2,2 \times 2$ ) excluded. For both addition and multiplication problems, small problems were defined as problems with a product smaller than 25 and large problems as problems with a product larger than 25 . The order of operation was counterbalanced for all grades. For second graders only, the addition and multiplication test were administered on two consecutive days, so as to keep the total session load manageable. For fourth and sixth graders both operations succeeded each other immediately. For each operation, five 
practice trials were presented to let the children get used to the task and the material.

The problems were presented one at a time in the centre of a computer screen. A trial started with a fixation point for $500 \mathrm{~ms}$. Then the problem was presented horizontally in Arabic format as dark-blue characters on a light-grey background, with the operation sign $(+$ or $\times)$ at the fixation point. Children were asked to verbally state their answer as soon as they knew it. The problem remained on screen until the child responded. Timing began when the stimulus appeared and ended when the response triggered the soundactivated relay. To enable this sound-activated relay, children wore a microphone which was activated when they spoke their answer aloud. This microphone was connected to a software clock accurate to $1 \mathrm{~ms}$. The experimenter entered the answer by means of the numerical path of the keyboard. On each trial, visual feedback was presented to the children, a happy face (smiley) when their answer was correct, and a sad face when their answer was incorrect.

Immediately after having solved the problem, children were asked to report verbally which strategy they had used to solve the problem. Taking into account the literature on strategy use in simple arithmetic, a distinction was made between three levels of strategies, namely Retrieval, Transformation, and Counting. Retrieval was explained as "remembering or knowing the answer directly from memory". If children said that the answer "just popped into their head", their strategy was coded as retrieval. Transformation was explained as "deriving the answer from some known facts" Examples were given, such as making an intermediate step to 10 (e.g., $8+5=8+2+3 ; 9 \times 4=10 \times 4-4$ ) and using a tie in order to solve a non-tie problem (e.g., $6+7=6+6+1 ; \quad 5 \times 6=5 \times 5+5)$. Counting was explained as "step-by-step counting to get the answer. For addition, this meant counting one-by-one, e.g., $4+3=4 \ldots 5 \ldots 6 \ldots 7$. No distinction was made between finger counting, counting all, or counting from the larger addend. For multiplication, counting meant (subvocally) reciting the multiplication tables, e.g., $8 \times 3=8 \ldots 16 \ldots 24$. A fourth category 'Other' was added to cover the case when the children used another strategy or did not know which strategy they had used. All incorrect trials and all trials that were corrupted due to failure of the voice activated relay were repeated at the end of the block to decrease the amount of data loss.

\section{Reading-span task}

This is a complex working-memory span task, in which materials (i.e. words) have to be maintained in memory while other information (i.e., sentences for comprehension) has to be processed. This task differs from simple shortterm memory tasks in which small amounts of materials have to be maintained and recalled without any processing load being imposed. The reading-span task (Daneman \& Carpenter, 1980) is a classical example of a complex working-memory task. In this task, participants have to read sets of increasing numbers of sentences aloud while retaining words in memory. Our Dutch reading-span task (see also De Jonge \& De Jong, 1996) included two practice trials (consisting of two sentences) and two trials for each consecutive number of sentences (range: $2-7$ sentences). The sentences were presented on a sheet of paper one-by-one and the child had to read them aloud. At the end of each sentence, a word was given by the experimenter, which had to be stored in memory. At the end of each sentence set, the child had to reproduce all words in the order in which they had been presented by the experimenter. As the number of sentences increased, the number of words-to-retain increased as well. For example, if the child had read 4 sentences after which each time a word was provided, the correct response after having read all the sentences consisted of 4 words. If the child failed at remembering the words in two sets with the same number of sentences/words, the reading-span task was stopped. The score on this task was the number of correctly remembered words (range: 4-54 words).

\section{Results}

Of all trials, $13 \%$ was spoiled due to failure of the soundactivated relay. Since all these invalid trials returned at the end of the block, most of them were recovered from data loss, which reduced the trials due to failure of the soundactivated relay to $3 \%$. Further, all trials on which children had used a strategy of the 'Other' category $(0.3 \%)$ were deleted. Finally, all the response times (RTs) more than 3 standard deviations from each participant's mean (per operation) were discarded as outliers (2\%).

The results section is divided into two parts. First, analyses of variance (ANOVAs) were carried out to investigate age-related effects on strategy selection and strategy efficiency; problem size and operation taken into account. Second, regression analyses were performed to test whether working-memory span plays a role in children's simplearithmetic strategy use. All reported results are considered to be significant if $P<0.05$, unless mentioned otherwise.

Analyses of variance

\section{Strategy selection}

The children used several strategies to solve the simplearithmetic problems. Not all strategies were chosen equally frequently, however. As can be seen in the upper panel of 
Fig. 1 Percentages of the used strategies for each grade (left second grade, middle fourth grade, right sixth grade), as a function of operation and problem size

\section{Addition}

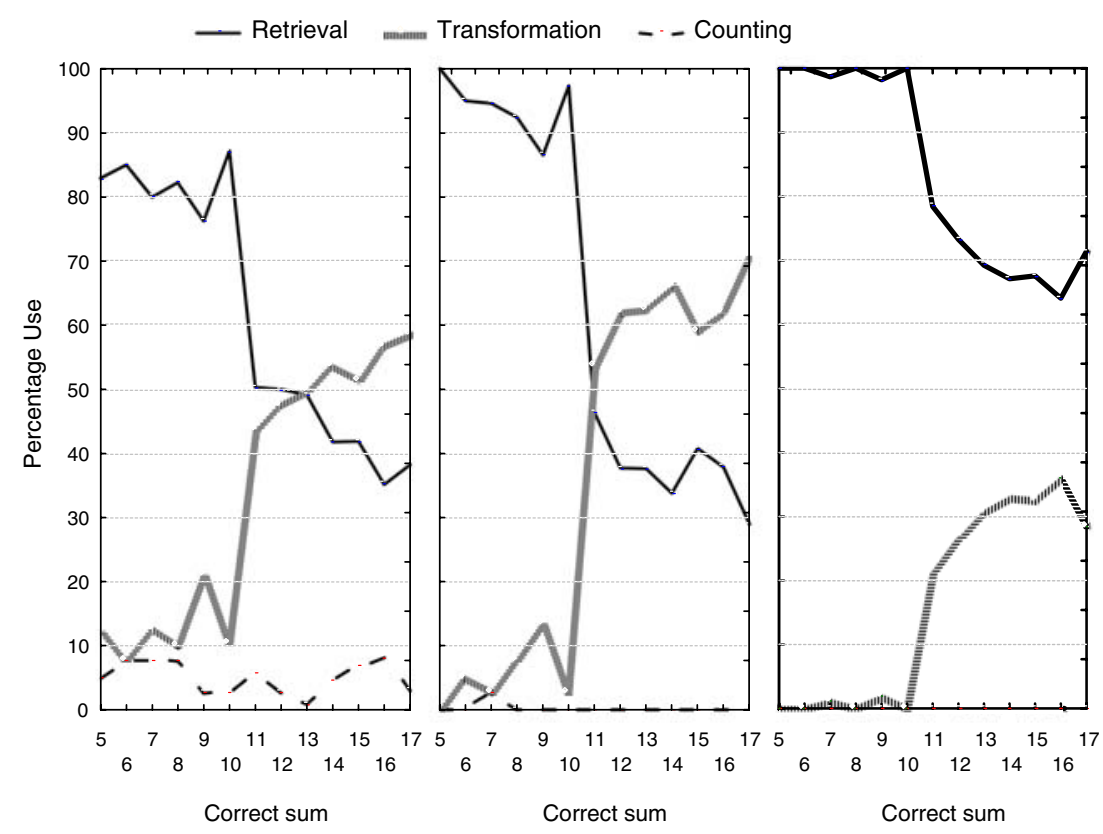

Multiplication

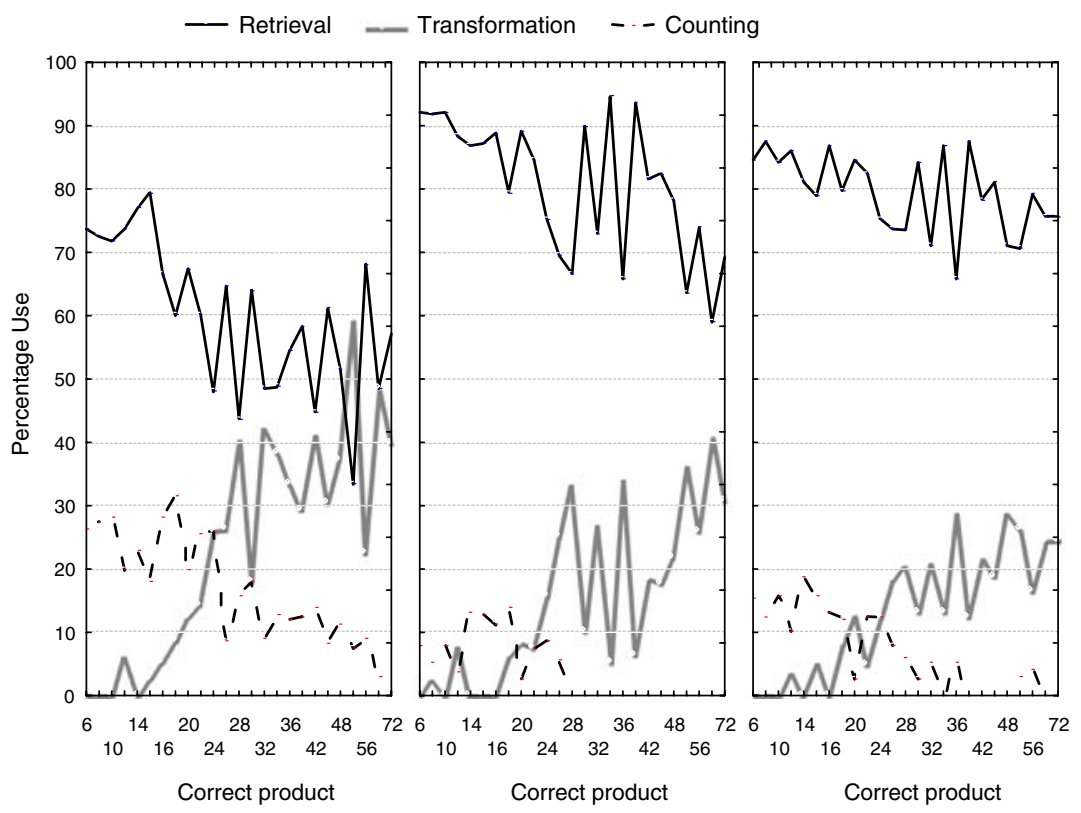

Fig. 1, children of all grades most often chose retrieval to solve addition problems with sums smaller than 10. As soon as the sum was larger than 10 , a tremendous decrease in retrieval use was observed. The second and fourth graders chose the transformation strategy more often than direct memory retrieval on problems with a solution above 10 . For the sixth graders, in contrast, retrieval was the most frequently used strategy to solve addition problems with sums both smaller and larger than 10. Analyses on the subject level showed that only eleven children used retrieval on all addition problems, two second graders, one fourth grader and eight sixth graders.

The lower panel of Fig. 1 shows the strategy choice pattern for multiplication problems. Here, retrieval use tended to decrease linearly with increasing problem size, whereas the frequency of transformation increased as problem size became larger. Another striking difference with the strategy choices for addition problems is that direct memory retrieval was the most popular strategy already from second grade on and on all problem sizes. Analyses on the subject 
Table 1 Mean percentages retrieval use for second, fourth, and sixth graders as a function of operation and problem size

\begin{tabular}{lllll}
\hline & & Second grade & Fourth grade & Sixth grade \\
\hline Addition & Small & $79(3)$ & $88(3)$ & $97(3)$ \\
& Large & $45(8)$ & $37(8)$ & $71(8)$ \\
\multirow{4}{*}{ Multiplication } & Small & $64(6)$ & $85(6)$ & $81(6)$ \\
& Large & $56(6)$ & $76(6)$ & $78(6)$ \\
\hline
\end{tabular}

Standard errors are shown between brackets

level showed that fourteen children used retrieval on all multiplication problems, one second grader, two fourth graders and eleven sixth graders. The very infrequent use of counting (in both addition and multiplication) was probably due to the curriculum in Belgium, which strongly advices children against using this strategy.

A 3 (Grade: 2, 4, 6) $\times 2$ (Problem size: small vs. large) $\times 2$ (Operation: addition vs. multiplication) ANOVA with repeated measures on problem size and operation was conducted on percentages of retrieval use (see Table 1). The main effect of grade was significant, $F(2,57)=4.6$, $M S_{e}=8625$. The percentage of retrieval use increased linearly across second, fourth and sixth grade, $F(1,57)=9.2$. We first tested whether this effect differed across small and large problems. Obviously, retrieval was more frequently used on small than on large problems, $F(1,57)=94.6$, $M S_{e}=299$. Although this effect of problem size was true in all grades $[F(1,57)=28.7,58.9$, and 14.5 , for second, fourth, and sixth grade, respectively], problem size interacted with grade, $F(2,57)=3.8$. The age-related trends in retrieval use thus differed as a function of problem size. More specifically, the increase in retrieval use between the second and the fourth grade was only significant for small problems, $F(1,57)=7.9$, and not for large problems, $F(1,57)<1$, whereas the increase in retrieval use between the fourth and the sixth grade was only significant for large problems, $F(1,57)=3.9$, and not for small problems, $F(1,57)<1$. Consequently, the difference in retrieval use between small and large problems (i.e., percentage of retrieval use on large problems - percentage of retrieval use on small problems) did not decrease between second and fourth rade $(P>0.10)$, but did decrease significantly between fourth and sixth grade, $F(1,57)=7.5$.

We then tested whether the increase in retrieval use differed across operations. Although the main effect of Operation did not reach significance, $F(1,57)=1.6$, the operation $\mathrm{x}$ grade interaction did, $F(2,57)=5.1$. Between second and fourth grade, there was a significant increase in retrieval use for multiplication problems, $F(1,57)=6.1$, and not for addition problems, $F(1,57)<1$, whereas the reverse was true between fourth and sixth graders, with a significant increase in retrieval use for addition problems,
$F(1,57)=8.6$, and not for multiplication problems, $F(1,57)<1$. This age-related pattern also explains why percentages of retrieval use did not differ across addition and multiplication problems in second graders, $F(1,57)<1$ : retrieval was used as frequently for both operations. Percentages retrieval use did not differ across operations in sixth graders either, with very frequent retrieval use on both addition problems and multiplication problems, $F(1,57)<1$. In the fourth grade, however, percentages retrieval use were larger on multiplication problems than on addition problems, $F(1,57)=11.1$.

\section{Retrieval efficiency: latency}

A 3 (Grade: 2, 4, 6) $\times 2$ (Problem size: small vs. large) $\times 2$ (Operation: addition vs. multiplication) ANOVA with repeated measures on problem size and operation was conducted on correct retrieval $\mathrm{RTs}^{2}$ (see Table 2a). The main effect of grade was significant, $F(2,57)=65.2$, $M S_{e}=1380585$. Retrieval RTs linearly decreased as children were older, as confirmed by a planned comparison with linear contrast, $F(2,57)=113.3$. We first tested whether this increase in retrieval efficiency differed between small and large problems. The main effect of problem size was significant, $F(1,57)=116.6, M S_{e}=225,284$, with faster retrieval on small than on large problems. Moreover, the retrieval problem-size effect (retrieval RTs on large problems-retrieval RTs on small problems) was significant in all grades, $F(1,57)=146.2,15.7$, and 7.0 for second, fourth and sixth grade, respectively. Grade interacted with problem size, however, $F(2,57)=26.1$. This interaction showed that the retrieval problem-size effect decreased significantly between second and fourth grade children $F(1,57)=33.0$, but did not differ between fourth and sixth grade children, $F(1,57)<1$. More precisely, between second and fourth grade, children became faster in retrieving large problems, whereas between fourth and sixth grade, they became slightly faster in retrieving both small and large problems.

We then tested whether the increase in retrieval efficiency differed across operations. Retrieval was faster on addition problems than on multiplication problems, $F(1,57)=6.2, M S_{e}=448058$, but operation did not interact with grade. The age-related increase in retrieval efficiency thus runs parallel for addition and multiplication.

\footnotetext{
${ }^{2}$ Since (a) not all children used both retrieval and procedural strategies, and (b) only RTs of the correctly solved problems were analyzed, for some children empty cells occurred in the ANOVAs on latencies. We replaced these empty cells for each child with the correct RT of the corresponding cell [i.e., the mean RT (over participants) of the grade $\mathrm{x}$ problem size $\mathrm{x}$ operation cell].
} 
Table 2 Mean retrieval and procedural latencies for second, fourth, and sixth graders as a function of operation and problem size

\begin{tabular}{lllll}
\hline & & Second grade & Fourth grade & Sixth grade \\
\hline Retrieval & & & & \\
Addition & Small & $2,252(113)$ & $1,195(113)$ & $966(113)$ \\
& Large & $3,806(261)$ & $1,682(261)$ & $1,328(261)$ \\
Multiplication & Small & $2,844(130)$ & $1,454(130)$ & $1,177(130)$ \\
& Large & $3,856(142)$ & $1,809(142)$ & $1,378(142)$ \\
Procedural & & & & \\
Addition & Small & $3,172(248)$ & $1,547(248)$ & $2,096(248)$ \\
& Large & $5,089(265)$ & $2,027(265)$ & $2,446(265)$ \\
Multiplication & Small & $5,786(548)$ & $3,139(548)$ & $3,525(548)$ \\
& Large & $7,854(484)$ & $2,939(484)$ & $4,033(484)$ \\
\hline Standard
\end{tabular}

Standard errors are shown between brackets

Table 3 Mean accuracies for second, fourth, and sixth graders as a function of operation and problem size

\begin{tabular}{lllll}
\hline & & Second grade & Fourth grade & Sixth grade \\
\hline Retrieval & & & & \\
Addition & Small & $98(1)$ & $99(1)$ & $100(1)$ \\
& Large & $95(1)$ & $98(1)$ & $98(1)$ \\
Multiplication & Small & $95(1)$ & $99(1)$ & $98(1)$ \\
& Large & $96(1)$ & $97(1)$ & $95(1)$ \\
Procedural & & & & \\
Addition & Small & $99(1)$ & $95(1)$ & $100(1)$ \\
& Large & $87(3)$ & $98(3)$ & $100(3)$ \\
Multiplication & Small & $86(2)$ & $98(2)$ & $99(2)$ \\
& Large & $78(4)$ & $95(4)$ & $97(4)$ \\
\hline
\end{tabular}

Standard errors are shown between brackets

\section{Retrieval efficiency: accuracy}

An Arcsin transformation was applied to the proportions of correct solutions. The same $3 \times 2 \times 2$ ANOVA was conducted on these Arcsin transformed values. To enhance comprehension however, Table $3 \mathrm{a}$ depicts percentages of correct answers. The main effect of grade did not reach significance, $F(2,57)=1.9$, but planned comparisons showed a significant increase in retrieval accuracy between second and fourth grade, $F(1,57)=3.6$, and no difference between fourth and sixth grade, $F(1,57)<1$. Small problems were retrieved significantly more accurately than large problems, $F(1,57)=9.8$, and this effect did not change across age [i.e., no grade $\mathrm{x}$ problem size interaction, $F(2,57)<1$ ]. There was also a trend towards higher accuracies on addition problems than on multiplication problems, $F(1,57)=3.3$ with $P=.07$, but this effect did not change across age either [i.e., no grade $\mathrm{x}$ operation interaction, $F(2,57)<1]$.

\section{Procedural efficiency: latency}

The same $3 \times 2 \times 2$ ANOVA was conducted on correct procedural RTs (see Table $2 \mathrm{~b}$ and footnote 2 ). The main effect of grade was significant, $F(2,57)=29.8$, $M S_{e}=6,988,988$. The second graders were significantly slower than fourth and sixth graders, $F(1,57)=53.0$ and $F(1,57)=34.2$, respectively, whereas there was no difference between fourth and sixth graders, $F(1,57)=2.0$. We first tested whether this age-related effect differed across small and large problems. Obviously, procedures were executed faster on small than on large problems, $F(1,57)=$ 38.9, $M S_{e}=1,093,316$. Problem size interacted with grade, though, $F(2,57)=18.3$ : the procedural problem-size effect (procedural RTs on large problems-procedural RTs on small problems) decreased significantly between second and fourth grade children, $F(1,57)=31.9$, but did not differ between fourth and sixth grade children, $F(1,57)<1$. Between second and fourth grade, children became faster in executing procedures on large problems, which reduced the problem-size effect. Consequently, the procedural problemsize effect was significant in second grade, $F(1,57)=71.9$, but not in fourth and sixth grade.

We also tested whether the increase in procedural efficiency differed across operations. The main effect of operation was significant, with higher procedural efficiencies on addition problems than on multiplication problems, $F(1,57)$ =52.5, $M S_{e}=3820986$. Operation also interacted with grade, $F(2,57)=3.0$. Planned comparisons showed that the increase in procedural efficiency was larger for multiplication problems than for addition problems between second and fourth grade, $F(1,57)=5.3$, but did not differ across operations between fourth and sixth grade, $F(1,57)<1$.

\section{Procedural efficiency: accuracy}

The same $3 \times 2 \times 2$ ANOVA was conducted on the Arcsin transformations of proportions of correct answers. Percentages of correct answers are shown in Table $3 \mathrm{~b}$. The main effect of grade was significant, $F(2,57)=13.5$. A planned comparison confirmed that procedural accuracies increased linearly with grade, $F(1,57)=24.7$. We first tested whether this age-related effect differed across small and large problems. Accuracies were higher on small than on large problems, $F(1,57)=9.8$. Furthermore, problem size interacted with grade, $F(2,57)=4.5$. Whereas the procedural problem-size effect (accuracy on large problems-accuracy on small problems) decreased significantly between second and fourth grade, $F(1,57)=8.4$, it did not change anymore between fourth and sixth grade, $F(1,57)<1$. Consequently, the procedural problem-size effect was significant in second grade, $F(1,57)=17.2$ but not in fourth and sixth grade (both $F \mathrm{~s}<1.5$ ). 
We then tested whether the increase in procedural efficiency differed across operations. Accuracies were higher on addition problems than on multiplication problems, $F(1,57)=14.6$. Operation also interacted with grade, $F(2,57)=3.1$. Addition accuracies did not increase between second and fourth grade, but did increase between fourth and sixth grade, $F(1,57)=4.8$. Multiplication accuracies, in contrast, increased between second and fourth grade, $F(1,57)=15.7$, but not between fourth and sixth grade, $F(1,57)<1$.

\section{Summary}

Analyses of variance were run to answer two questions formulated in the introduction. Concerning the first question (Are all three sources of the problem-size effect present in children and do they change across age?), results showed that two sources of the problem-size effect were present in all grades, namely more frequent retrieval use on small than on large problems, and more efficient retrieval use on small than on large problems. The third source of the problemsize effect, more efficient procedural use on small than on large problems, was only present in the second grade. Moreover, the different sources of the problem-size effect changed across age: The decrease in the size of the problem-size effect was first (i.e., between second and fourth grade) due to more efficient retrieval use and more efficient procedural use, after which (i.e., between fourth and sixth grade) it was due to more frequent retrieval use.

Concerning the second question (Does children's simple-arithmetic strategy use differ between addition and multiplication, and does this difference change across age?), results showed that the improvement in strategic performance (more frequent retrieval use and more efficient procedural use) on multiplication was especially apparent between second and fourth grade. The improvement in strategic performance on addition, in contrast, was especially apparent between fourth and sixth grade. Finally, the agerelated improvement in retrieval efficiency was equally large for addition and multiplication. In the next section, regression analyses are run to test the role of workingmemory span in children's simple-arithmetic strategy use across the primary school years (cfr. our third research question).

\section{Regression analyses}

Before presenting the results of the regression analyses, we first report children's performance on the working-memory span task, which is one of the predictors used in the regression analyses. Children's performance on the workingmemory span task was tested with an ANOVA with Grade $(2,4,6)$ as between-subjects factor. As expected, the main effect of Grade was significant, $F(2,57)=37.8$, with increasing working-memory span across the second, fourth, and sixth grade (scores of 12.7, 20.2, and 28.0, corresponding to working-memory spans of $2.4,3.3$, and 4.2 , respectively). The question now is whether complex workingmemory span plays a role in strategy selection or strategy efficiency. More precisely, we will test whether workingmemory span predicts variance in percentages of retrieval use, retrieval latencies, and procedural latencies. To this end, correlation and regression analyses were conducted for each dependent variable (i.e., retrieval use, retrieval latency, and procedural latency ${ }^{3}$ separately.

Retrieval use was regressed on working-memory span, problem size ${ }^{4}$, and operation. Retrieval latency and procedural latency were regressed on the same three variables and on percentage retrieval use as well. Doing so, we wanted to test Siegler's (1988) prediction that retrieval frequency should correlate with retrieval efficiency but not with procedural efficiency. Indeed, both retrieval frequency and retrieval efficiency are in his model of strategy choice dependent on the peakedness of problem-answer associations, whereas procedural efficiency depends on the difficulty of executing the particular procedure. Correlation results can be found in Table 4 and regression results can be found in Table 5 .

\section{Retrieval use}

The percentage of retrieval use was regressed on problem size, operation, and working-memory span, for each grade separately. For second graders, the total amount of variance explained $\left(R^{2}\right)$ was $.073, F(3,778)=20.36$. Retrieval use was significantly more frequent on small than on large problems, and for high-span children than for low-span children. Operation was not significantly predictive. For the fourth grade, $R^{2}=.088$ and $F(3,1056)=34.12$, smaller problem sizes predicted more frequent retrieval use, whereas working-memory span did not play a significant role. Direct memory retrieval was also more frequently used on multiplication problems than on addition problems. For the sixth grade finally, $R^{2}=.013$ and $F(3,975)=4.45$, retrieval use was more frequent on small than on large

\footnotetext{
${ }^{3}$ As age-related differences were substantially smaller in accuracy data than in latency data (cfr. ANOVA results), regression analyses were performed on latency data only.

${ }^{4}$ In the regression analyses, problem size was determined by the correct answer of the problem (i.e., sizes from 5 to 17 for addition problems and sizes from 6 to 72 for multiplication problems). Thus, whereas a dichotomous measure of problem size was used in the analyses of variance, a continuous measure of problem size was used in the regression analyses. Operation was in the regression analyses coded by a dummy variable with value 1 for addition problems and value -1 for multiplication problems.
} 
Table 4 Correlations between retrieval use, retrieval RTs, procedural RTs, problem size, operation, and working-memory span

* $P$ is significant at the 0.01 level (2-tailed)

Table 5 Summary of the regression analyses for variables predicting percentage retrieval use, retrieval RTs, and procedural RTs

*P is significant at the 0.01 level

\begin{tabular}{llllll}
\hline & Retrieval RT & Procedural RT & Problem size & Operation & WM span \\
\hline Second grade & & & & & \\
Retrieval use & $-0.239^{*}$ & $-0.164^{*}$ & $-0.138^{*}$ & 0.017 & $0.218^{*}$ \\
Retrieval RT & & $0.445^{*}$ & $0.344^{*}$ & $-0.159^{*}$ & $-0.174^{*}$ \\
Procedural RT & & $0.293^{*}$ & $-0.163^{*}$ & $-0.284^{*}$ \\
Fourth grade & & & & \\
Retrieval use & $-164^{*}$ & 0.057 & -0.018 & $-0.224^{*}$ & -0.045 \\
Retrieval RT & & $0.547^{*}$ & $0.402^{*}$ & $-0.212^{*}$ & $-0.224^{*}$ \\
Procedural RT & & & $0.342^{*}$ & $-0.315^{*}$ & $-0.285^{*}$ \\
Sixth grade & & & & & \\
Retrieval use & $-0.320^{*}$ & -0.132 & $-0.102^{*}$ & 0.021 & 0.022 \\
Retrieval RT & & $0.303^{*}$ & $0.251^{*}$ & $-0.116^{*}$ & -0.035 \\
Procedural RT & & & $0.412^{*}$ & $-0.253^{*}$ & $0.228^{*}$ \\
\hline
\end{tabular}

\begin{tabular}{|c|c|c|c|c|c|c|c|c|c|}
\hline & \multicolumn{3}{|c|}{ Second grade } & \multicolumn{3}{|c|}{ Fourth grade } & \multicolumn{3}{|c|}{ Sixth grade } \\
\hline & $B$ & SE & $\beta$ & $B$ & SE & $\beta$ & $B$ & SE & $\beta$ \\
\hline \multicolumn{10}{|l|}{ Retrieval use } \\
\hline Problem size & -0.50 & 0.11 & $-0.194 *$ & -0.64 & 0.10 & $-0.237^{*}$ & -0.33 & 0.09 & $-0.140^{*}$ \\
\hline Operation & -3.48 & 1.70 & -0.087 & -15.15 & 1.52 & $-0.366^{*}$ & -2.33 & 1.47 & -0.063 \\
\hline WM span & 2.03 & 0.32 & $.218 *$ & -0.31 & 0.20 & -0.044 & 0.12 & 0.18 & .022 \\
\hline \multicolumn{10}{|l|}{ Retrieval RTs } \\
\hline Problem size & 47.74 & 6.19 & $0.354 *$ & 19.03 & 1.88 & $0.388^{*}$ & 9.64 & 1.42 & $0.270^{*}$ \\
\hline Operation & 42.00 & 93.21 & 0.021 & -2.36 & 29.70 & -0.003 & 24.87 & 21.79 & 0.045 \\
\hline WM span & -75.13 & 18.55 & $-0.153 *$ & -27.68 & 3.91 & $-0.218^{*}$ & -0.54 & 2.61 & -0.007 \\
\hline Retrieval use & -15.82 & 3.07 & $-0.196 *$ & -4.95 & 1.24 & $-0.125^{*}$ & -13.22 & 1.36 & $-0.312 *$ \\
\hline \multicolumn{10}{|l|}{ Procedural RTs } \\
\hline Problem size & 86.27 & 15.38 & $0.302 *$ & 22.65 & 5.66 & $0.252 *$ & 39.43 & 7.32 & $0.387 *$ \\
\hline Operation & 198.2 & 237.4 & -0.045 & -195.8 & 92.04 & -0.134 & -94.96 & 116.1 & -0.059 \\
\hline WM span & -302.3 & 46.47 & $-0.272 *$ & -69.36 & 11.01 & $-0.284 *$ & 63.38 & 15.73 & $0.241 *$ \\
\hline Retrieval use & -14.52 & 6.57 & -0.094 & -6.86 & 2.47 & -0.126 & -3.51 & 3.41 & -0.062 \\
\hline
\end{tabular}

problems, whereas no effects of operation or working-memory span were observed. Note that operation was only significantly predictive of percentage retrieval use in fourth graders, which fits well with the ANOVA on retrieval use.

\section{Retrieval efficiency}

Retrieval latencies were regressed on problem size, operation, working-memory span, and percentage retrieval use, for each grade separately. The $R^{2}$ was .192 for the second grade, $F(4,585)=34.73, R^{2}=.226$ for the fourth grade, $F(4,819)=59.77$ and $R^{2}=.162$ for the sixth grade, $F(4,818)=39.66$. Problem size was significantly predictive in all grades, with faster retrieval use on small than on large problems. The percentage retrieval use was significantly predictive in all grades as well, with faster retrieval use when retrieval was more frequently used. Working- memory span was only predictive in second and fourth grade, with faster retrieval use for high-span than for lowspan children. Operation was not predictive in any grade.

\section{Procedural efficiency}

Procedural latencies were regressed on problem size, operation, working-memory span, and percentage retrieval use, for each grade separately. The $R^{2}$ was 0.174 for the second grade, $F(4,475)=25.03, R^{2}=.216$ for the fourth grade, $F(4,392)=27.04$ and $R^{2}=0.238$ for the sixth grade, $F(4,227)=17.71$. Problem size was significantly predictive in all grades, with more efficient procedural strategy execution on small than on large problems. Working-memory span was predictive in all grades as well, but the relation between span and procedural efficiency changed across grades. High-span second and fourth graders were more 
efficient procedural strategy users than were low-span second and fourth graders, but high-span sixth graders were less efficient procedural strategy users than were low-span sixth graders. Span-related differences in strategy selection can explain this unexpected result. Indeed, retrieval was used more frequently in high-span sixth graders (85\%) than in low-span sixth graders $(78 \%)$, and this difference was larger for large problems (79\% vs. 69\%) than for small problems (91\% vs. $87 \%$ ). Consequently, high-span sixth graders used procedural strategies to solve the largest problems only, which results in large procedural RTs in high-span sixth graders. Indeed, procedural RTs for large problems were larger for high-span than for low-span sixth graders (3,707 ms vs. $3,089 \mathrm{~ms}$, respectively), whereas procedural RTs for small problems did not differ between highspan and low-span sixth graders $(2,772 \mathrm{~ms}$ vs. $2,533 \mathrm{~ms}$, respectively). Operation and percentage retrieval did not predict procedural efficiency in any grade.

\section{Summary}

The advantage of having a large working-memory span decreased across grades, especially regarding retrieval frequency and retrieval efficiency. More specifically: (1) working-memory span significantly predicted retrieval frequency for second graders but not for fourth and sixth graders, and (2) working-memory span predicted retrieval efficiency for second and fourth graders but not for sixth graders. Comparably, the execution of procedural strategies benefited from a high working-memory span in second and fourth grade only. Because high-span sixth graders used procedural strategies almost exclusively on large problems, procedural efficiency decreased for these children. Strategy efficiency data were thus influenced by the children's strategy choices. This bias can be avoided by using the choice/ no-choice method (Siegler \& Lemaire, 1997), as discussed further in this paper. A final interesting observation was that, as predicted by Siegler (1988), percentage retrieval use did predict retrieval efficiency but not procedural efficiency.

\section{General discussion}

Children's arithmetic strategic performance increased with age: older children used memory retrieval more often, were faster and more accurate in retrieving arithmetic facts, and were faster and more accurate in executing procedural strategies. In the remaining of this chapter, we discuss whether or not (and from which moment on) children's arithmetic strategy use resembles adults' arithmetic strategy use. We successively discuss the problem-size effect, operationrelated effects, and the role of working-memory span. The discussion section ends with an evaluation of the present results within a model of arithmetic strategic performance.

The problem-size effect

From fourth to sixth grade, retrieval use increased for large problems but not for small problems. As such, the problemsize effect caused by strategy selection processes became smaller from fourth grade on. However, the retrieval strategy was more frequently used on small than on large problems in all age groups. Strategy selection processes were thus a significant source of the problem-size effect in second, fourth, and sixth graders. From second to fourth grade, retrieval and procedural efficiencies increased for large problems but not for small problems. This way, the problem-size effect caused by strategy efficiency processes became smaller from second grade on. However, the retrieval problem-size effect (i.e., the difference in retrieval RTs between large and small problems) stayed significant in all grades. The procedural problem-size effect (i.e., the difference in procedural RTs between large and small problems), in contrast, was significant in second grade only. Thus, whereas problem size affected retrieval frequency and retrieval efficiency in all age groups, problem size affected procedural efficiency in second grade only.

Importantly, previous studies showed that all three sizerelated effects on strategy use (i.e., less frequent retrieval use for large than for small problems, lower retrieval efficiency for large than for small problems, and lower procedural efficiency for large than for small problems) are significant sources of the problem size effect in adults (e.g., Campbell \& Xue, 2001). The first two factors were significant in the current child study as well. Concerning the third factor, something strange occurred: the procedural problem-size effect was present in second grade but disappeared in fourth and sixth grade. However, it reappeared in secondary-school children (Imbo et al., 2007, in press). The proficiency for solving small and large problems equally efficiently by means of procedures is probably caused by practice and schooling effects. As soon as children finish elementary school, such effects disappear, resulting in less efficient procedure execution, especially for large problems. Comparable effects have been reported by Geary (1996), who observed that the problem-size effect disappeared and reversed between 1st and third grade Chinese children, but re-appeared in Chinese adults. The investigation of the appearance, disappearance, and re-appearance of the problem-size effect across lifetime provides interesting ideas for future research.

To conclude, the decreasing problem-size effect was associated with an increase in strategy efficiency for younger children and with an increase in retrieval frequency for older children. Moreover, the size-related effect on strategy 
efficiency did not change anymore from fourth grade on. Since De Brauwer et al. (2006) observed that the problemsize effect remains equally large from sixth grade on till adulthood; we might conclude that children from fourth grade on have developed a memory network that strongly resembles the adult memory network. This conclusion is in agreement with previous studies which maintain that mental-arithmetic networks might be completely operational from third grade on (e.g., Ashcraft \& Fierman, 1982; Koshmider \& Ashcraft, 1991; Lemaire, Barrett, Fayol, \& Abdi, 1994).

\section{Differences between addition and multiplication}

Children of all age groups retrieved addition facts more efficiently (i.e., faster and more accurately) than they retrieved multiplication facts. Comparable effects have been observed in adults (e.g. Campbell \& Xue, 2001; Hecht, 1999; Imbo et al., 2007, in press). Importantly, the main effect of operation in retrieval efficiency (i.e., more efficient retrieval use for addition than for multiplication) did not change across the primary school years. This indicates consistent changes in the speed with which addition and multiplication facts are retrieved from long-term memory. Thus, although the addition and multiplication network may differ across age groups in general (i.e., main differences in retrieval speed), their development seems to run fairly parallel (i.e., no interaction between age and operation). Otherwise stated, addition and multiplication involve similar retrieval processes across childhood (this study) and in adulthood (e.g., Campbell \& Oliphant, 1992; Geary, Widaman, \& Little, 1986; Miller, Perlmutter, \& Keating, 1984).

Another persistent effect in adults is that retrieval is used more frequently in multiplication than in addition (e.g. Campbell \& Xue, 2001; Hecht, 1999; Imbo et al. 2007, in press). Surprisingly, in the current study, this operationrelated effect on strategy selection was significant in fourth grade only. In second grade, where children only start to learn the multiplication tables, retrieval was used as frequently in addition as in multiplication. However, multiplication fact retrieval significantly increased from second to fourth grade, an effect that was probably due to the great emphasis of the Belgian school system on the memorization of multiplication tables. Because the amount of retrieval use in multiplication reached in fourth grade (81\%) was comparable to that observed in Belgian adults (73-88\%; Imbo et al., 2007, in press; Imbo \& Vandierendonck, 2007) and in North-American adults (64$88 \%$, Campbell \& Xue, 2001), there was no space left for another increase in retrieval use. Thus, for multiplication, children from fourth grade on may already have developed a complete memory network that strongly resembles an adult network (see also De Brauwer et al., 2006). For addition, retrieval use still increased from fourth to sixth grade. This effect should be attributed to general practice effects (e.g., increases in processing speed) rather than to specific training effects (e.g., explicit memorization of addition facts).

Finally, procedural efficiency was higher in addition than in multiplication, an effect observed in adults as well (e.g. Campbell \& Xue, 2001; Hecht, 1999; Imbo et al., 2007 , in press). All age groups showed higher efficiencies in addition than in multiplication and this effect boosted from fourth grade on. Indeed, between second and fourth grade, procedural efficiency increased for multiplication but not for addition. This early increase in multiplication efficiency might be related to two facts. First, multiplication is heavily trained from second grade on. As noted above, children are taught to solve multiplication problems very fast and accurately. Obviously, increases in procedural efficiency are the precursors of increases in retrieval use. Second, multiplication strategy efficiency is more susceptible to change than addition strategy efficiency. In a previous study, we observed that adults' daily arithmetic experience (e.g., the amount of arithmetic lessons in secondary school) influenced multiplication efficiency but not addition efficiency (Imbo et al., 2007, in press). Moreover, when explicitly practicing arithmetic problems, effects were larger in multiplication efficiency than in addition efficiency (Imbo \& Vandierendonck, 2007). These effects can be accounted for by the relative efficiency of procedural strategies for addition and multiplication. Indeed, in both children and adults, addition strategies are far more efficient (i.e., faster) than multiplication strategies. Consequently, it is less demanding to increase multiplication efficiencies than to increase addition efficiencies. Strategy selection and strategy efficiency processes in other arithmetic operations (such as subtraction and division) have been investigated less frequently (but see Campbell \& Xue, 2001; Imbo \& Vandierendonck, 2007b, c; Robinson et al., 2006; Seyler et al., 2003) and are an issue for further research.

\section{The role of working memory}

Working-memory span was related to strategy selection in second grade only. High-span second graders used retrieval more frequently than did low-span second graders, but this effect disappeared in fourth and sixth graders. Up until now, the relevance of working memory in children's strategy selection process was equivocal, since some studies observed a correlation between working-memory span and retrieval use (e.g., Barrouillet \& Lépine, 2005), whereas others did not (e.g., Geary et al., 2004). The current study suggests that strategy selection processes tend to rely on working-memory resources in young children only. This 
runs parallel to adult studies which observed that working memory is not needed in strategy selection (e.g., Hecht, 2002; Imbo \& Vandierendonck, 2007b, c).

Working-memory span was related to retrieval efficiency in second and fourth grade, with less efficient retrieval use in low-span children than in high-span children. Less efficient retrieval use in low-span children than in highspan children has been observed earlier (e.g., Barrouillet \& Lépine, 2005). Apparently, low-span children develop flatter distributions of problem-answer associations, resulting in less frequent and less efficient retrieval use. However, with growing age and increasing practice and schooling, even low-span children can develop peaked distributions of problem-answer associations. Consequently, the differences between low-span children and high-span children decrease across primary school years. In adults, the role of working-memory in strategy efficiency has been investigated by means of dual-task studies rather than correlational studies. The evidence is equivocal: Hecht (2002) observed no effects of working-memory load on retrieval efficiency whereas Imbo and Vandierendonck (2007b, c) did observe less efficient fact retrieval under working-memory load. Future research is needed to specify the relation between working memory and direct fact retrieval in both children and adults.

Finally, procedural efficiency was also related to working-memory span. In second and fourth grade, low-span children executed procedural strategies less efficiently than did high-span children. A significant role of working memory in procedural strategy execution has been observed in adults as well (e.g., Hitch, 2002; Imbo et al., 2007b, c). The role of working memory in procedural strategies is quite obvious: Each procedure requires several subprocesses that require working-memory resources, such as storing intermediate results, keeping track of several steps, integrating information, et cetera (see DeStefano \& LeFevre, 2004, for a review). High-span children and adults can carry out these various subprocesses with fewer demands on a limited resource pool than low-span children and adults. Consequently, high-span children have more working-memory resources left for storage while processing the problem, resulting in higher procedural efficiency scores.

Surprisingly, we observed higher procedural efficiencies in low-span sixth graders than in high-span sixth graders. We suppose that this effect was due to an artifact. Indeed, high-span sixth graders used procedural strategies to solve the largest problems only, which might have increased their procedural RTs relative to low-span sixth graders, who used procedural strategies on smaller problems as well. This artifact occurred because we only used a choice condition, in which strategy efficiency data are biased by strategy selection effects (Siegler \& Lemaire, 1997). Such a bias would have been avoided by using the choice/no-choice method (devised by Siegler \& Lemaire, 1997), which not only entails a choice condition, but also no-choice conditions. In no-choice conditions, participants are asked to use one single strategy to solve all problems. In a recent study using the choice/no-choice method, we indeed showed that loading sixth graders' working-memory resources resulted in less efficient procedural strategy use (Imbo \& Vandierendonck, 2007a).

Finally, it should be noted that the advantage of having a large working-memory capacity decreased across age. The relation between working-memory span and retrieval frequency was not significant anymore from fourth grade on, and the relation between working-memory span and retrieval efficiency was not significant anymore from sixth grade on. These results are consistent with Ackerman's (1988) findings. Specifically, working memory is most important during the initial phase of arithmetic-skill acquisition and its role declines as procedures are used less frequently and facts become represented in long-term memory. Working-memory resources might thus be needed to achieve a complete representation of number facts in long-term memory (e.g., Geary, 1990; Geary \& Brown, 1991; Hitch \& McAuley, 1991; Siegler \& Shrager, 1984), which explains the correlation between working-memory span and retrieval use in the younger children. However, once the number facts are completely represented in longterm memory, fact retrieval becomes more automatic and less effortful, resulting in smaller arithmetic-performance differences between high-span children and low-span children.

A model of strategic change

In the ASCM (Siegler \& Shipley, 1995), outlined in the introduction, people have several strategies available and try to choose the best one. Strategy selection occurs on the basis of knowledge on each strategy's efficiency. Each time a simple-arithmetic problem is solved correctly, the problem-answer association increases, resulting in a more peaked distribution of problem-answer associations. The more peaked the distribution of problem-answer associations, the more frequently retrieval is used; while the use of procedural strategies vanishes. This reasoning fits with our data, since the frequency of retrieval use increased across age.

Across age, the efficiency of both retrieval and procedural strategies increased as well. This observation can also be accounted for by the ASCM. Indeed, each time an answer is retrieved from long-term memory, the problemanswer association is strengthened. As outlined above, this results in more peaked distributions of problem-answer associations and thus in more efficient retrieval use. Each execution of a procedural strategy brings an increase in the 
strategy's speed and a decrease in its probability of generating an error. The ASCM thus accounts for age-related increases in both retrieval efficiency and procedural efficiency.

To summarize, in the ASCM, both retrieval frequency and retrieval efficiency depend on the peakedness of the problem-answer association, whereas procedural efficiency does not. We tested the hypothesis that the frequency of retrieval use would be highly correlated with retrieval efficiency but not with procedural efficiency. Regression results confirmed this prediction, since the frequency of retrieval use was highly predictive of retrieval efficiency in all grades, whereas the frequency of retrieval use was not predictive of procedural efficiency.

In the following, we verify whether the ASCM is able to account for the size-related, operation-related, and resource-related results observed in children and adults. First, what does the ASCM tell about the problem-size effect? Because small problems are more frequently encountered, young children develop peaked problemanswer associations for small problems and relatively flat problem-answer associations for large problems. They might also set larger search lengths for small problems, because they are taught that small problems should be retrieved in any case. More peaked problem-answer associations and larger search lengths for small problems than for large problems results in increases in retrieval frequency for small problems but not in increases in retrieval efficiency for small problems, which is exactly what we observed between second and fourth grade. However, as children grow older, the emphasis shifts towards large problems. This results in more peaked problem-answer associations for large problems and thus in more frequent retrieval use for large problems, as observed between fourth and sixth grade.

However, some results are more difficult to explain by the ASCM. Theoretically, the ASCM predicts that extensive practice should create equally peaked problem-answer associations for small and large problems. Accordingly, retrieval frequency and retrieval efficiency should be equal in small as in large problems. Such effects, however, have not yet been observed; the retrieval problem-size effect is still present in adults (e.g., Campbell \& Xue, 2001; Imbo et al., in press; LeFevre et al., 1996a, b), even after explicit practice (e.g., Imbo \& Vandierendonck, 2007). One explanation for the persisting problem-size effect is that adult's problem-answer associations for small problems are still stronger for small problems than for large problems. Another explanation is based on interference effects (Campbell, 1987, 1995): answers to large problems undergo stronger interference effects than do answers to small problems, resulting in less efficient retrieval for large than for small problems. Future research is needed to clear up the persistent nature of the problem-size effect across lifetime.

Second, what can the ASCM tell about the differences across addition and multiplication? More frequent retrieval use for multiplication than for addition suggests more peaked distributions of associations for multiplication than for addition. However, multiplication facts are retrieved less efficiently than addition facts, which suggests rather the opposite (i.e., more peaked distributions for addition than for multiplication). Thus, although the notion of peaked and flat distributions is relevant within each operation (i.e., peaked distributions predict frequent and fast retrieval use), it is not clear how this notion can account for differences in retrieval frequency and retrieval speed across operations. One possibility is that the search length is larger for multiplication than for addition. Indeed, this would result in more frequent retrieval use for multiplication than for addition and in less efficient (i.e., slower) retrieval use for multiplication than for addition. The more efficient procedural use for addition than for multiplication can easily be accounted for by the ASCM, in which procedural efficiencies are based on the amount and the difficulty of steps. Because fewer increments of counting steps are needed in addition procedures than in multiplication procedures, procedural strategies are much easier to implement in addition than in multiplication.

Finally, we consider whether the ASCM may account for the role of working memory in strategic performance. Although working memory is not explicitly included in the ASCM, it has been predicted (e.g., Geary, 1993, 1994) that low-span children use slow counting procedures, which lead to delays in problem encoding and consequently to weak problem-answer associations and flat distributions of associations. High-span children, in contrast, develop strong problem-answer associations and more peaked distributions of associations, resulting in more frequent and more efficient retrieval use. More frequent and more efficient retrieval use in high-span children than in lowspan children is exactly what we observed. However, with growing age and experience, even low-span children may develop strong problem-answer associations and more peaked distributions of associations. Hence, the differences in retrieval performance between low-span and high-span children should decrease across the primary school years, as was observed in the current study.

It should be noted, however, that other models may also account for the relation between working-memory span and retrieval performance. The time-based resource-sharing model of working memory (Barrouillet et al., 2004), for example, predicts that lower working-memory resources reduce the amount of attentional resources available to activate knowledge from long-term memory (see also Cowan, 1999; Lovett et al., 1999). Consequently, poor working- 
memory resources not only impair the formation of associations in long-term memory but also the retrieval of existing associations. In other models (e.g., Engle, 2001; Engle, Kane, \& Tuholski, 1999), lower working-memory resources reduce the ability to resist interference, which might also result in less efficient retrieval performance. Thus far, our and other's results (e.g., Barrouillet \& Lépine, 2005) do not contradict the theories mentioned above. Future research is thus needed to investigate the predictive value of several models in the domain of mental arithmetic and cognitive strategy use, and more specifically to investigate the specific role of working memory in retrieval frequency and retrieval efficiency.

Acknowledgments The research reported in this article was supported by grant no. 011D07803 of the Special Research Fund at Ghent University to the first author and by grant no. 10251101 of the Special Research Fund of Ghent University to the second author. Thanks are extended to the elementary school "Gemeentelijke basisschool" in Koksijde (Belgium), where all experiments were administered, and to David Geary and Mark Ashcraft for their helpful comments on previous drafts of this article.

\section{References}

Ackerman, P. L. (1988). Determinants of individual differences during skill acquisition: Cognitive abilities and information processing. Journal of Experimental Psychology: General, 117, 288-318.

Adams, J. W., \& Hitch, G. J. (1997). Working memory and children's mental addition. Journal of Experimental Child Psychology, 67, 21-38.

Ashcraft, M. H., \& Fierman, B. A. (1982). Mental addition in 3rd, fourth, and sixth graders. Journal of Experimental Child Psychology, 32, 216-234.

Ashcraft M. H. (1992) Cognitive arithmetic: A review of data and theory. Cognition, 44, 75-106.

Baddeley, A. D. (1996). Exploring the central executive. Quarterly Journal of Experimental Psychology, 49A, 5-28.

Baddeley, A. D., \& Logie, R. H. (1999). Working memory: The multicomponent model. In A. Miyake, \&P. Shah (Eds) Models of working memory: Mechanisms of active maintenance and executive control (pp. 28-61). New York: Cambridge University Press.

Barrouillet, P., Bernardin, S., \& Camos, V. (2004). Time constraints and resource sharing in adults' working memory spans. Journal of Experimental Psychology: General, 133, 83-100.

Barrouillet, P., \& Lépine, R. (2005). Working memory and children's use of retrieval to solve addition problems. Journal of Experimental Child Psychology, 91, 183-204.

Campbell, J. I. D. (1987). Network interference and mental multiplication. Journal of Experimental Psychology: Learning, Memory, \& Cognition, 13, 109-123.

Campbell, J. I. D. (1995). Mechanisms of simple addition and multiplication: A modified network-interference theory and simulation. Mathematical Cognition, 1, 121-164.

Campbell, J. I. D., \& Graham, D. J. (1985). Mental multiplication skill - Structure, process, and acquisition. Canadian Journal of Psychology, 39, 338-366.

Campbell J. I. D., \& Oliphant, M. (1992). Representation and retrieval of arithmetical facts: A network-interference model and simulation. In J. I. D. Campbell (ed.), The nature and origins of mathematical skills (pp. 331-364). Amsterdam: Elsevier.
Campbell, J. I. D., \& Xue, Q. (2001). Cognitive arithmetic across cultures. Journal of Experimental Psychology: General, 130, 299-315.

Cooney, J. B., Swanson, H. L., \& Ladd, S. F. (1988). Acquisition of mental multiplication skill; Evidence for the transition between counting and retrieval strategies. Cognition and Instruction, 5, 323-345.

Cowan, N. (1999). An embedded-process model of working memory. In A. Miyake, \& P. Shah (Eds.), Models of working memory: Mechanisms of active maintenance and executive control (pp. 62101). Cambridge, UK: Cambridge University Press.

Daneman, M., \& Carpenter, P. A. (1980). Individual differences in working memory and reading. Journal of Verbal Learning and Verbal Behavior, 19, 450-466.

De Brauwer, J., Verguts, T., \& Fias, W. (2006). The representation of multiplication facts: Developmental changes in the problem size, five and tie effects. Journal of Experimental Child Psychology, $94,43-56$.

De Jonge, P., \& De Jong, P. (1996). Working memory, intelligence and reading ability in children. Personality and Individual Differences, 21, 1007-1020.

DeStefano, D., \& LeFevre, J.-A. (2004). The role of working memory in mental arithmetic. European Journal of Cognitive Psychology, $16,353-386$.

De Vos, T. (1992). Tempo Test Rekenen [Arithmetic Tempo Test]. Lisse, Switserland: Swets and Zeitlinger.

Engle, R. W. (2001). What is working memory capacity? In H. L. Roediger III, J. S. Nairne, I. Neath, \& M. Surprenant (Eds.), The nature of remembering: Essays in honor of Robert G. Crowder (pp. 297314). Washington, DC: American Psychology Association.

Engle, R. W., Kane, M. J., \& Tuholski, S. W. (1999). Individual differences in working memory capacity, what they tell us about controlled attention, general fluid intelligence, and functions of the prefrontal cortex. In A. Miyake \& P. Shah (Eds.), Models of working memory: Mechanisms of active maintenance and executive control (pp. 102-134). Cambridge, UK: Cambridge University Press.

Geary, D. C. (1990). A componential analysis of an early learning deficit in mathematics. Journal of Experimental Child Psychology, 49, 363-383.

Geary, D. C. (1993). Mathematical disabilities: Cognitive, neuropsychological and genetic components. Psychological Bulletin, 114, 345-362.

Geary D. C. (1994). Children's mathematical development: Research and practical applications. Washington, DC: American Psychological Association.

Geary D. C. (1996). The problem-size effect in mental addition: Developmental and cross-national trends Mathematical Cognition, 1996, 63-93.

Geary, D. C. (2004). Mathematics and learning disabilities. Journal of Learning Disabilities, 37, 4-15.

Geary, D. C., \& Brown, S. C. (1991). Cognitive addition - Strategy choice and speed-of-processing differences in gifted, normal, and mathematically disabled-children. Developmental Psychology, 27, 398-406.

Geary, D. C., Bow-Thomas, C. C., Liu, F., \& Siegler, R. (1996). Development of arithmetical competencies in Chinese and American children: Influence of age, language, and schooling. Child Development, 67, 2022-2044.

Geary, D. C., Hoard, M. K., Byrd-Craven, J., \& DeSoto, M. C. (2004). Strategy choices in simple and complex addition: Contributions of working memory and counting knowledge for children with mathematical disability. Journal of Experimental Child Psychology, 88, 121-151.

Geary, D. C., Widaman, K. F., \& Little, I. D. (1986). Cognitive addition and multiplication: Evidence for a single memory network. Memory \& Cognition, 14, 478-487. 
Hecht, S. A. (1999). Individual solution processes while solving addition and multiplication facts in adults. Memory \& Cognition, 27, 1097-1107.

Hecht, S. A. (2002). Counting on working memory in simple arithmetic when counting is used for problem solving. Memory and Cognition, 30, 447-455.

Hitch, G. J., \& McAuley, E. (1991). Working memory in children with specific arithmetical difficulties. British Journal of Psychology, $82,375-386$.

Hopkins, S. L., \& Lawson, M. J. (2002). Explaining the acquisition of a complex skill: Methodological and theoretical considerations uncovered in the study of simple addition and the moving-on process. Educational Psychology Review, 14, 121-154.

Imbo, I., \& Vandierendonck, A. (2007a). The development of strategy use in elementary-school children: Working memory and individual differences. Journal of Experimental Child Psychology (in press).

Imbo, I., \& Vandierendonck, A. (2007b). The role of phonological and executive working-memory resources in simple-arithmetic strategies. European Journal of Cognitive Psychology (in press).

Imbo, I., \& Vandierendonck, A. (2007c) Do multiplication and division strategies rely on executive and phonological working-memory resources? Memory \& Cognition (in press).

Imbo, I., \& Vandierendonck, A. (2007). Practice effects on strategy selection and strategy efficiency in simple mental arithmetic. Manuscript submitted for publication.

Imbo, I., Vandierendonck, A., \& Rosseel, Y. (2007). The influence of problem features and individual differences on strategic performance in simple arithmetic. Memory \& Cognition (in press).

Jurden, F. H. (1995). Individual differences in working-memory and complex cognition. Journal of Educational Psychology, 87, 93102.

Koshmider, J. W., \& Ashcraft, M. H. (1991). The development of children's mental multiplication skills. Journal of Experimental Child Psychology, 51, 53-89.

LeFevre, J.-A., Bisanz, J., Daley, K. E., Buffone, L., Greenham, S. L., \& Sadesky, G. S. (1996a). Multiple routes to solution of single-digit multiplication problems. Journal of Experimental Psychology: General, 125, 284-306.

LeFevre, J.-A., Sadesky, G. S., \& Bisanz, J. (1996b). Selection of procedures in mental addition: Reassessing the problem size effect in adults. Journal of Experimental Psychology: Learning, Memory, \& Cognition, 22, 216-230.

Lemaire, P., Barrett, S. E., Fayol, M., \& Abdi, H. (1994). Automatic activation of addition and multiplication facts in elementary school children. Journal of Experimental Child Psychology, 57, 224-258.

Lépine, R., Roussel, J. L., \& Fayol, M. (2003). Procedural resolution or direct retrieval in memory of simple addition and multiplication in children? Année Psychologique, 103, 51-80.

Lovett, M. C., Reder, L. M., \& Lebière, C. (1999). Modeling working memory in an unified architecture: An ACT-R perspective. In: A.
Miyake, \& P. Shah (Eds.), Models of working memory: Mechanisms of maintenance and executive control (pp. 135-182). Cambridge, UK: Cambridge University Press.

Miller, K., Perlmutter, M., \& Keating, D. (1984). Cognitive arithmetic: Comparison of operations. Journal of Experimental Psychology, $10,46-60$.

Noël, M. P., Seron, X., \& Trovarelli, F. (2004). Working memory as a predictor of addition skills and addition strategies in children. Current Psychology of Cognition, 22, 3-25.

Rasmussen, C., \& Bisanz, J. (2005). Representation and working memory in early arithmetic. Journal of Experimental Child Psychology, 91, 137-157.

Robinson, K. M., Arbuthnott, K. D., Rose, D., McCarron, M. C., Globa, C. A., \& Phonexay, S. D. (2006). Stability and change in children's division strategies. Journal of Experimental Child Psychology, 93, 224-238.

Roussel, J. L., Fayol, M., \& Barrouillet, P. (2002). Procedural vs direct retrieval strategies in arithmetic: A comparison between additive and multiplicative problem solving. European Journal of Cognitive Psychology, 14, 61-104.

Seyler, D. J., Kirk, E. P., \& Ashcraft, M. H. (2003). Elementary subtraction. Journal of Experimental Psychology: Learning, Memory, and Cognition, 29, 1339-1352.

Siegler, R. S. (1988). Strategy choice procedures and the development of multiplication skill. Journal of Experimental Psychology: General, 117, 258-275.

Siegler, R. S., \& Lemaire, P. (1997). Older and younger adults' strategy choices in multiplication: Testing predictions of ASCM using the choice/no-choice method. Journal of Experimental Psychology: General, 126, 71-92.

Siegler R. S., \& Shipley, C. (1995). Variation, selection, and cognitive change. In T. J. Simon, \& G. S. Halford (Eds.), Developing cognitive competence: New approaches to process modeling (pp. 31-76). Hillsdale, NJ: Lawrence Erlbaum.

Siegler, R. S., \& Shrager, J. (1984). Strategy choices in addition, subtraction: How do children know what to do? In C. Sophian (Eds.), Origins of cognitive skills (pp. 229-294). Hillsdale, NJ: Erlbaum.

Steel, S., \& Funnell, E. (2001). Learning multiplication facts: A study of children taught by discovery methods in England. Journal of Experimental Child Psychology, 79, 37-55.

Swanson, H. L. (2004). Working memory and phonological processing as predictors of children's mathematical problem solving at different ages. Memory \& Cognition, 32, 648-661.

Swanson, H. L., \& Beebe-Frankenberger, M. (2004). The relationship between working memory and mathematical problem solving in children at risk and not at risk for serious math difficulties. Journal of Educational Psychology, 96, 471-491.

Zbrodoff, N. J. (1995). Why is $9+7$ harder than $2+3$ ? Strength and interference as explanations of the problem-size effect. Memory \& Cognition, 23, 689-700. 